\title{
Aplicación de las matemáticas en marketing para las empresas
}

Aplicación de las matemáticas en marketing para las empresas

Aplicação da matemática em marketing para empresas

\section{ARTÍCULO ORIGINAL}

$\begin{array}{ccc}\text { Lita Elvira Saldaña Dávila } & \text { Edin Becerra Celiz } & \text { Teresa Juliana Jara Alarcón } \\ \text { 1saldanad@unc.edu.pe } & \text { ebecerrac@unach.edu.pe } & \underline{\text { teresa.jara@unh.edu.pe }} \\ \underline{\text { https://orcid.org/0000-0001-8688-9522 }} & \underline{\text { https://orcid.org/0000-0001-8270-2628 }} & \underline{\underline{\text { https://orcid.org/0000-0001-7464-9361 }}} \\ \begin{array}{c}\text { Universidad Nacional de Cajamarca } \\ \text { Cajamarca, Perú }\end{array} & \begin{array}{c}\text { Universidad Nacional Autónoma de Chota } \\ \text { Cajamarca, Perú }\end{array} & \begin{array}{c}\text { Universidad Nacional de Huancavelica } \\ \text { Huancavelica, Perú }\end{array}\end{array}$

Recibido 05 de Febrero 2021 | Arbitrado y aceptado 19 de Abril 2021 | Publicado en 28 Julio 2021

\section{RESUMEN}

Las empresas generalmente, como toda organización, se ven cada vez enfrentadas a nuevos desafíos y retos para conservar su postura competitiva en el mercado. Los consumidores, clientes y clientes potenciales poseen accesos a información como jamás: por ende, las organizaciones se hallan con un mercado invadido de información. Frente a esta ola de datos, las organizaciones tienen que estar preparadas para la acertada toma de decisiones, fundamentada en indagaciones de mercado debidamente estructuradas y analizadas. Las matemáticas constituyen un instrumento de gran costo para los primordiales ejecutivos de una compañía, puesto que tienen la posibilidad de usarla como un recurso para la toma de decisiones en el marketing. Es pertinente producir una cultura matemática en el centro de las empresas.

Palabras clave: Matemáticas, investigación de mercados, decisiones de marketing.

\begin{abstract}
Companies generally, like any organization, are increasingly faced with new challenges and challenges to maintain their competitive position in the market. Consumers, clients and potential clients have access to information like never before: therefore, organizations find themselves with a market invaded by information. Faced with this wave of data, organizations have to be prepared for correct decisionmaking, based on properly structured and analyzed market inquiries. Mathematics is an instrument of great cost for the main executives of a company, since they have the possibility of using it as a resource for decisionmaking in marketing. It is pertinent to produce a mathematical culture in the center of companies.
\end{abstract}

Keywords: Mathematics, market research, marketing decisions.

\section{RESUMO}

As empresas em geral, como qualquer organização, enfrentam cada vez mais novos desafios e desafios para manter sua posição competitiva no mercado. Consumidores, clientes e potenciais clientes têm acesso à informação como nunca antes: por isso, as organizações encontram-se com um mercado invadido pela informação. Diante dessa onda de dados, as organizações precisam estar preparadas para a correta tomada de decisões, com base em pesquisas de mercado devidamente estruturadas e analisadas. A matemática é um instrumento de grande custo para os principais executivos de uma empresa, pois eles têm a possibilidade de utilizá-la como recurso para a tomada de decisão em marketing. É pertinente produzir uma cultura matemática no centro das empresas.

Palavras-chave: Matemática, pesquisa de mercado, decisões de marketing. 


\section{Introducción}

El estudio de las transformaciones ocurridas en los intercambios comerciales ha manifestado la necesidad de implementar nuevas estrategias que permitan el alcance los objetivos planteados para cada organización. En este sentido, muchas empresas hacen sus esfuerzos para lograr llegar al cliente y hacerse asiduo de su aceptación, confianza y aprobación, para que en el orden de las ideas publicitarias los productos sean consumidos de una manera efectiva satisfaciendo las de necesidades de los clientes y generando mayor valor en ellos.

En este orden de ideas, muchas compañías hacen uso de estrategias de marketing con la finalidad de crear nuevas campañas publicitarias que ofrezcan al consumidor una oferta tentativa para que los productos sean adquiridos. Estas estrategias de marketing se dedican a la investigación de las características del mercado, de modo tal que los ofrecimientos encajen en sus necesidades cuya finalidad es absorber, persuadir, mantener y fidelizar a los clientes, y de esta manera satisfacer sus intereses o resolver sus problemas.

Ahora bien, concretando los temas de marketing, pueden encontrarse una gran cantidad de variables que intervengan en el proceso del estudio de mercados y que precisamente ayudan a tomar decisiones en cuanto a la estructura publicitaria que debe asumirse en un determinado momento, sin embargo, vale destacar una que se considera de gran relevancia para la implementación de nuevos métodos de comercialización y venta de productos, se trata de la influencia de las matemáticas en las campañas de marketing.

El uso de las matemáticas en el marketing va más allá de un simple cálculo de ingresos y egresos, de inversiones, de ganancias y pérdidas, no se trata solamente de aplicar formulas y ofrecer resultados sino de conocer de manera adecuada el significado de cada valor para tomar las decisiones acertadas, es decir, la información derivada de la aplicación de modelos matemáticos abre un abanico de posibilidades para insertar productos o marcas en espacios pertinentes con el objetivo de reconocer las variaciones de necesidad, intereses y gustos de consumo de los usuarios, con ello se puede prever a desaciertos en con base a estadísticas, hacer balances entre ella capacidad de las marcas, explorar nuevos segmentos, analizar otros mercados y potenciar el conocimiento mediante las interacciones digitales, entre otros.

Este artículo tuvo como objetivo explorar la relación existente entre las matemáticas y el marketing, como una alternativa metodológica para la toma de 
decisiones. Se realizó una revisión sistemática de la literatura científica con base en investigaciones y artículos desarrollados en el área administrativa y en cuanto al proceso de búsqueda y recojo referente a los datos, seleccionándose descriptores de búsqueda como: modelos matemáticos, marketing y las 7P de marketing, en idioma español.

En la temática del marketing, se hace mención a elementos externos e internos que determinan las decisiones al momento de ofrecer un producto de acuerdo a las características del público objetivo y en torno a ello, Empresariales (2019) sostiene que se trata de la combinación de distintas estrategias que se aplican como implementación del marketing tomando en cuenta todos los factores que conforman un estudio de mercado, donde se destacan el producto, precio, promoción, plaza, persona, proceso y presentación.

En referencia al producto, se considera todo lo relativo a la adjudicación de productos al consumidor, en ello, debe tenerse en cuenta las condiciones del mismo en el sentido de su estado al momento de su entrega, lo cual permite optimizar los recursos de tiempo y dinero ya que estas previsiones ayudan a fortalecer la eficiencia. Respecto al producto, Quiroa (2019) debe caracterizarse por presentar características diferenciales respecto a los que se encuentran en el mercado, ofreciendo ventajas atractivas a los clientes, de tal modo que pueda satisfacer las pretensiones de preferencias de los clientes.

Por su parte, para Empresariales (2019), el precio va a estar directamente relacionado con la identificación del cliente teniendo en cuenta los costos que genera su elaboración, distribución y comercialización, el promedio de ganancias, los métodos de pago, entre otros gastos, con la intención de ofrecer las mejores y más atractivas condiciones para atraer al cliente ya que al adquirir el producto, el cliente pagaría siempre y cuando vea cubiertas sus necesidades e intereses. Otro elemento que conforma esta estrategia de marketing es la promoción, la cual se emplea para dar a conocer los productos que se pretenden ofrecer, ello consiste en emplear publicidad, métodos de venta y promociones para mostrar las ventajas del producto y los beneficios de su consumo cuya fase consiste en la comunicación con el público objetivo para persuadirlo e informar sobre los beneficios del bien ofrecido cristalizado en la compra del mismo.

La plaza representa el lugar donde se presentan, fabrican, venden o distribuyen los productos, lo que significa que el conocimiento de este espacio permite el diseño de actividades que se adecuen a la comercialización visual del producto y con ello se presta facilidad para ser ubicado por el cliente. Esto declara la forma como la empresa ubica los productos en los lugares, los tiempos y los contextos más adecuados para el cliente, por 
cuanto el producto debe ajustarse a la necesidad de cada sujeto. La persona se refiere tanto a los vendedores como a los clientes. En este sentido, cuando el cliente se siente atendido satisfactoriamente, significa que la promoción de la marca ha sido exitosa y en ello se incluye la atención que se le ha proporcionado en el momento de la venta, por cuanto reclutar al personal adecuado es beneficioso para la empresa, en este sentido, todas las personas que trabajan en una empresa deben unificar sus esfuerzos para dar el mejor servicio al cliente, porque el cliente es su razón de ser.

Seguidamente se presenta el proceso, el cual contiene el conglomerado de actividades realizadas por la empresa en el sostenimiento de sus relaciones con los consumidores y que arroja los elementos necesarios para implementar medidas que aseguren la fidelización del cliente, en esta etapa se emplean los medios de información $\mathrm{y}$ datos automatizados, para dar una mejor respuesta a los consumidores y de esa manera servirles mejor, para seguir contando con su preferencia y su recompra. Por último, se menciona el posicionamiento que se conoce como la presencia, y amerita que se establezcan las acciones para mantener un lugar predominante en las preferencias de los usuarios, ello tiene mucho que ver con la imagen que tiene el público acerca de la marca y la pretensión es ocupar un lugar privilegiado en la mente del consumidor, para que nuestra marca sea recordada, reconocida y preferida.

\section{Metodología}

La metodología que se utilizó se basa en una revisión sistemática de papers, publicaciones periódicas, estudio de aplicativos recientes y portales web especializados para comprender como funciona, además el grado de progreso actualmente alcanzado, reconociendo cuáles son sus aplicaciones más importantes.

Con la intención de realizar la revisión de los estudios realizados asociados al tema se procedió a investigar publicaciones científicas de alto impacto a través de la consulta de bases de datos como Scopus, Google Académico y Proquest.

De igual manera, para la búsqueda antes mencionada se procedió a utilizar las palabras clave Matemáticas, investigación de mercados, decisiones de marketing; aplicándose durante la búsqueda la combinación de los descriptores entre sí a través de los operadores boléanos "and" y "or". Conjuntamente, se puso en práctica el uso de ciertos criterios o filtros de búsqueda como "artículos de revistas" para hacer más específica el acceso a manuscritos depositados en las diversas bases de datos examinadas. 
Los criterios de inclusión aplicados estuvieron asociados con la búsqueda a través de los filtros de búsqueda aplicados y las especificaciones antes nombradas, es decir que sean manuscritos de revistas científicas, con un máximo de vigencia de 5 años, y que el tema de búsqueda guarde estrecha relación con la temática y con las variables de Matemáticas, investigación de mercados, decisiones de marketing. Por lo tanto, fueron descartadas todos aquellos manuscritos que no estuvieran enmarcados en lo antes mencionado, así como aquellos que se consideraban deficientes o códigos DOI u URL que tenían enlaces averiados.

\section{Resultado y discusión}

\subsection{Marketing}

Desde finales del siglo XIX se ha puesto en práctica la expresión, siendo hasta inicio de siglo XX cuando se conoció la referencia académica originaria como tal, a través del científico Edward Jones para el año 1901 en su conferencia "The United States distribution and regulatory industry", (Bartels, 1988) y Hernández y Rodríguez (2003) en la University of Michigan aseveran que en el presente curso se enseñaba los diferentes tipologías de bienes de marketing, su categorización, grados y tipologías de marcas, de igual manera se hacía énfasis de las diferencias entre los comercios mayoristas y minoristas (Baines, Fill \& Page, 2011).

Es innegable la continua evolución del término Marketing a través del tiempo, observándose un consenso compañías y clientes, con una orientación más enfocada a los clientes y menos a métodos de fabricación (González, López \& Otero, 2014). En este orden de ideas, la American Marketing Association (en adalante, AMA) (2008) aplicaba una definición del concepto desarrollada en los años 30 conocida como "el resultado de las actividades empresariales que dirigen el flujo de bienes y servicios desde el productor hasta el consumidor". Sin embargo, Kotler \& Keller (2013) expone una visión más evolucionada, más actualizada orientada a los consumidores y a satisfacer sus necesidades, a través de la definición redactada como "un proceso social y administrativo mediante el cual grupos e individuos obtienen lo que necesitan y desean a través de generar, ofrecer e intercambiar productos de valor con sus semejantes".

En este orden de ideas, Kotler \& Keller (2013) instauran un nuevo proceso de marketing fundamentado en el estudio del mercado y su entorno, determinando los públicos objetivos y mercados a partir de la base del marketing mix, concepción 
anteriormente introducida por McCarthy en 1956 (Jobber, 2007), conformada por las populares en gestión empresarial 4 ps: Product, Place, Price \& Promotion.

Como se muestra, es fundamental que este término evolucione a través del tiempo para poder comprender con claridad esta disciplina en la actualidad. Por lo que, la AMA (2008) redefinir el marketing nuevamente como "la actividad realizada por un conjunto de instituciones y los procesos para crear, comunicar, entregar e intercam- biar ofertas que tienen valor para los consumidores, clientes, socios y la sociedad en general". De igual manera, se considera necesario comprender una de los principales aportes de Westwood (2013), que permite explicar el marketing moderno de una mejor manera, esto debido a que se refiere más a políticas de lealtad del cliente, redes sociales y CRM (Customer Relationship Management). De igual manera, no se puede obviar algunos factores claves como estrategia empresarial en general, y en particular la capacidad competitiva, señalada por Porter (2002), quien la precisa como una ventaja empresarial única, con capacidad de conservarse a través del tiempo, siendo mejor que la oferta que promete la competencia, haciéndola imposible de imitar, siendo además ajustable a una diversidad de escenarios de mercado.

Para Barney (2002) en el mundo del marketing una empresa puede crear ciertos atributos competitivos orientadas hacia el consumidor o por el valor de marca; incluso pueden ponerse en práctica una diversidad de criterios un poco más tangibles y objetivos como una calidad mejor del producto, producción más eficaz, con un desarrollo de estudios basados en la rentabilidad, recursos humanos bien tratados, ventajas en costes, entre otros.

Por tal razón, finalmente se puede exponer que, el marketing se puede definir como la "gestión de un proceso que identifica, define, crea, comunica, entrega y sostiene valor a clientes y grupos de interés, cumpliendo los objetivos de la organización" (Baker \& Hart, 2008), en líneas generales, también puede considerarse como la gestión de las variables y procesos que están inmersas dentro de la empresa u organización y las que se muestran hacia el exterior de la misma, direccionadas hacia el cliente, convirtiéndolo en un público fundamental, manteniendo siempre una visión basada en el beneficio económico si es el caso de empresas comerciales, o de beneficio social para otro tipo de entidades (Suárez, 2018; Dann, 2010). 


\subsection{P's del Marketing}

\subsubsection{Nociones básicas}

Según la Real Academia Española se considera como el conjunto de habilidades y prácticas que tienen como fin generar el crecimiento del comercio, fundamentalmente de la demanda.

Para Kotler, Philip, Armstrong y Gary (1998) es considerada como el trámite social y administrativo por medio del cual las personas y grupos adquieren lo que les hace falta y anhelan por medio de la generación e intercambio con otros de productos.

Stanton et al. (2000), lo mencionan como "un sistema total de actividades cuya finalidad es planear, fijar precio, promover y distribuir los productos satisfactores de necesidades entre los mercados meta para alcanzar los objetivos corporativos".

Kotler y Armstrong (2001), lo definen como la satisfacción generada al consumidor a través de un beneficio, donde el triunfo de la organización dependerá de su capacidad de cautivar clientes nuevos brindando beneficios superiores a los prometidos por sus competidores y conservar los existentes compensando sus anhelos y requerimientos.

Para Stanton, William J., Etzel, Michael J., Walker, Bruce J. (2007) este se describe como un sistema total de labores de negocios creado con la intención de generar productos que satisfagan sus, fijarles un costo, hacerles una promoción efectiva y proveerlos a los mercados objetivo, esto con la intención de alcanzar los objetivos de la organización. Este concepto presenta dos importantes implicaciones:

- $\quad$ Enfoque: todo el sistema de acciones de negocio debe estar encauzadas al cliente. Por lo que las necesidades de estos deben ser reconocidas y compensadas.

Duración: todo proceso de marketing debe iniciarse con una noción del producto que beneficiará al cliente y no culminar hasta cumplir con el completo benefício de estos, pudiendo ser que a través del tiempo sea cuando se haga el intercambio.

Debido al desarrollo progresivo de todos los servicios basados en una sociedad post- industrial se propuso la necesidad de replantear todas las definiciones y principios fundamentales asociados al marketing. De igual manera, en los años ochenta se destacó el aporte de Booms y Bitner a través del conocido marketing mix extendido; dichos investigadores en su propuesta lo definen mix por estar fundamentado en los 7Ps donde se adiciona a los anteriores cuatro elementos tres elementos conocidos como personas (participants), los componentes palpables (physicalevidence) y todos los procesos (process). Dichos elementos son indispensables según el tipo de servicio, considerándose 
a todas las personas que participan en el servicio, denominados como personal y beneficiarios; de igual manera todos aquellos componentes materiales asociados al servicio que van desde los que hacen referencia a las tecnologías; y los métodos que se encuentran asociados a la empresa que presta del servicio.

Para Booms y Bitner (1981), el concepto de gestión de marketing viene siendo el concepto de combinación de marketing (Mix Marketing). Cuyo término no es considerado una teoría de la gestión generada de un diagnóstico científico, siendo más bien considerada como un planteamiento conceptual donde se hace énfasis de principales tomas de decisiones del gerente de marketing con la intención de conformar ofertas con la intención de cumplir con los requerimientos de los consumidores. Por lo que estos investigadores definieron las "7Ps" de la mezcla de marketing como se observa seguidamente:

\section{Etapas de la Mercadotecnia}

Las actividades de la mercadotecnia se desarrollan en cualquier contexto, por lo que para analizar sus funciones estas deben ser divididas en tres fases o etapas conocidas como el medio, el proceso y la estrategia.

\section{Fase una o medio}

Conformado por todas aquellas acciones asociadas con el espacio donde se lleva a cabo el proceso de mercadotecnia, conocido como el Medio. Los datos pueden ser obtenidos a través de dos fuentes conocidas como el mercado (espacio donde se despliega el proceso de mercadotecnia) y el consumidor (quien recibirá los productos).

\section{Fase dos o proceso}

Aquí debe estructurarse todo aquel proceso que se debe tomar en cuenta para llevar a cabo todas las actividades, etapa que se conoce como el Proceso. En esta se experimentará la aplicación de las 7P de la mercadotecnia, popularmente conocidas como "mixtura".

\section{Fase tres o estrategia}

En esta fase se precisan todos los planes y gestiones que lleven al éxito, fase también conocida como la Estrategia; esta viene siendo realizada por los encargados de la administración, los cuales se encargan de llevar a cabo una diversidad de funciones entre las que se encuentran: asignación de obligaciones, propuesta de objetivos, establecimiento del nivel de alcance y sus restricciones y en desarrollo de un método de mercadotecnia con la intención de colocar el producto en lugares donde se garantice su consumo. 
Componentes de la Mercadotecnia

En la fase dos se analizarán las 7P de la mercadotecnia, siendo sus elementos los siguientes:

\section{Producto}

Incluye la descripción de las características del producto o servicio, tales como su presentación, su tamaño, su forma, su color, su olor, etc.

\section{Precio}

Indica el valor monetario que el comprador debe pagar para obtener el producto. Este valor se basa en lo siguiente: Costos fijos (materia prima y producción) + Costos variables (servicio, distribución, ventas, comunicación e investigación de mercado)+ Utilidad-Descuento $=$ Precio

\section{Plaza o distribución}

Se refiere al lugar físico (o virtual) en el cual se vende un producto, con lo años se prefiere hablar de distribución, debido a que el este concepto es más amplio e incluye los distintos canales o medios para hacer llegar el producto desde el fabricante, pasando por intermediarios, hasta llegar al consumidor final.

\section{Promoción}

Se refiere a todas las formas en las que una empresa comunica a su mercado las características y beneficios de los productos, se incluye aquí la publicidad.17

\section{Posventa o Servicio}

Son las actividades que efectúan el industrial y el comercializador, para garantizar que el producto es bueno y también para que regrese el consumidor.16

\section{Personas}

Hace referencia al personal que proporciona el servicio, por lo que las empresas deben preocuparse en reclutar, capacitar, motivar y retener a su personal de la mejor manera.

\section{Procesos}

Se refiere que para crear y entregar los elementos del servicio brindado a los clientes, se requiere el diseño y la implementación de procesos eficaces que describan el método y como se llevarán a cabo todas las acciones necesarias para que funcione el sistema al servicio del consumidor; por lo que es necesario tomar en cuenta ciertas características que serán realizadas las actividades, todas aquellas etapas que se consideren necesarias para se ejecuten los procesos ejecutar un proceso y que garantice la intervención del consumidor durante los procesos. 


\section{Evidencia Física}

Abarca todos los elementos tangibles que exhibe la empresa que presta el servicio, entre las que se pueden nombrar: estado de la fachada de los edificios, las zonas de jardines, la flota de vehículos, condiciones del mobiliario interior, todos los dispositivos, aspecto de personal, y todas aquellas señales visibles que muestren de manera tangible los servicios prestados por una compañía.

\subsection{Matemáticas en el marketing}

De acuerdo con las aseveraciones anteriores, es importante destacar que la Estadística Aplicada representa una herramienta de gran valía a la actividad desarrollada en las organizaciones, de hecho, su incorporación en el marketing empresarial es un hecho real en virtud de que se han asumido diversas resoluciones procedentes de los estudios de mercado que se optimizan al emplear adecuadamente la estadística. En torno a ello, es preciso tener en cuenta que mientras se incremente el uso de variables cuantitativas, las bondades de la estadística potencia en mayor medida los datos que conllevan a una toma de decisiones más acertada.

Los elementos que muestran el comportamiento de los elementos del mercado representan información valiosa para el marketing y, en consecuencia, marcan un camino para ser determinantes de las decisiones asumidas por los ejecutivos. En este orden de ideas, Gómez (2013) la estadística es un instrumento que permite disminuir los riesgos de equivocarse al tomar decisiones respecto a una situación dada.

Al hablar de estudios de mercados, también cabe especificar que, por ejemplo, los informes derivados de la estadística se relacionan íntimamente con la toma de decisiones, no obstante, su aplicación estará supeditada a los fines de la organización debido a que estos resultados determinarán las condiciones que responderán al objetivo mercado que persigue las investigaciones. Al respecto, Allen (2001) señala que la estadística puede ofrecer elementos que permitan establecer las aspiraciones y predilecciones de los usuarios respecto al nuevo producto.

Por todo ello, se indica que, dado que no se puede administrar lo que no se puede medir, el uso de Matemáticas y estadística en el Marketing son indispensables. De esta forma, se hace posible desarrollar el estudio de mercado, cuantificación de demanda, proyección de ventas, precios, rentabilidad. 


\subsubsection{Herramientas Matemáticas}

Algunas herramientas matemáticas para el marketing son, por ejemplo, los algoritmos. Estos tienen un rol trascendental en el futuro del desarrollo del marketing, ya que en la actualidad asumen diversas actividades propias de esta industria y paralelamente sirven de guía para la toma de decisiones estratégicas.

En ese sentido, los Algoritmos de árbol de decisión, por ejemplo, establecen un sistema de preguntas y llevan a cabo una evaluación de probabilidades de éxito/fracaso o si/no para cada decisión. También cabe mencionar a los Algoritmos de regresión (o lineal), los cuales realizan una interrelación entre distintas variables para así poder predecir el valor más apropiado y evitar errores. Los Algoritmos Bayesianos, por su parte, utilizan el teorema de probabilidad de Bayes para resolver cualquier problema y clasificar respecto a la probabilidad.

En tanto, los algoritmos genéticos son técnicas de inteligencia artificial que hacen posible hallar soluciones para una diversidad de problemas. Los tres tipos de operadores del Algoritmo Genético son: Selección o reproducción; Cruce; Mutación.

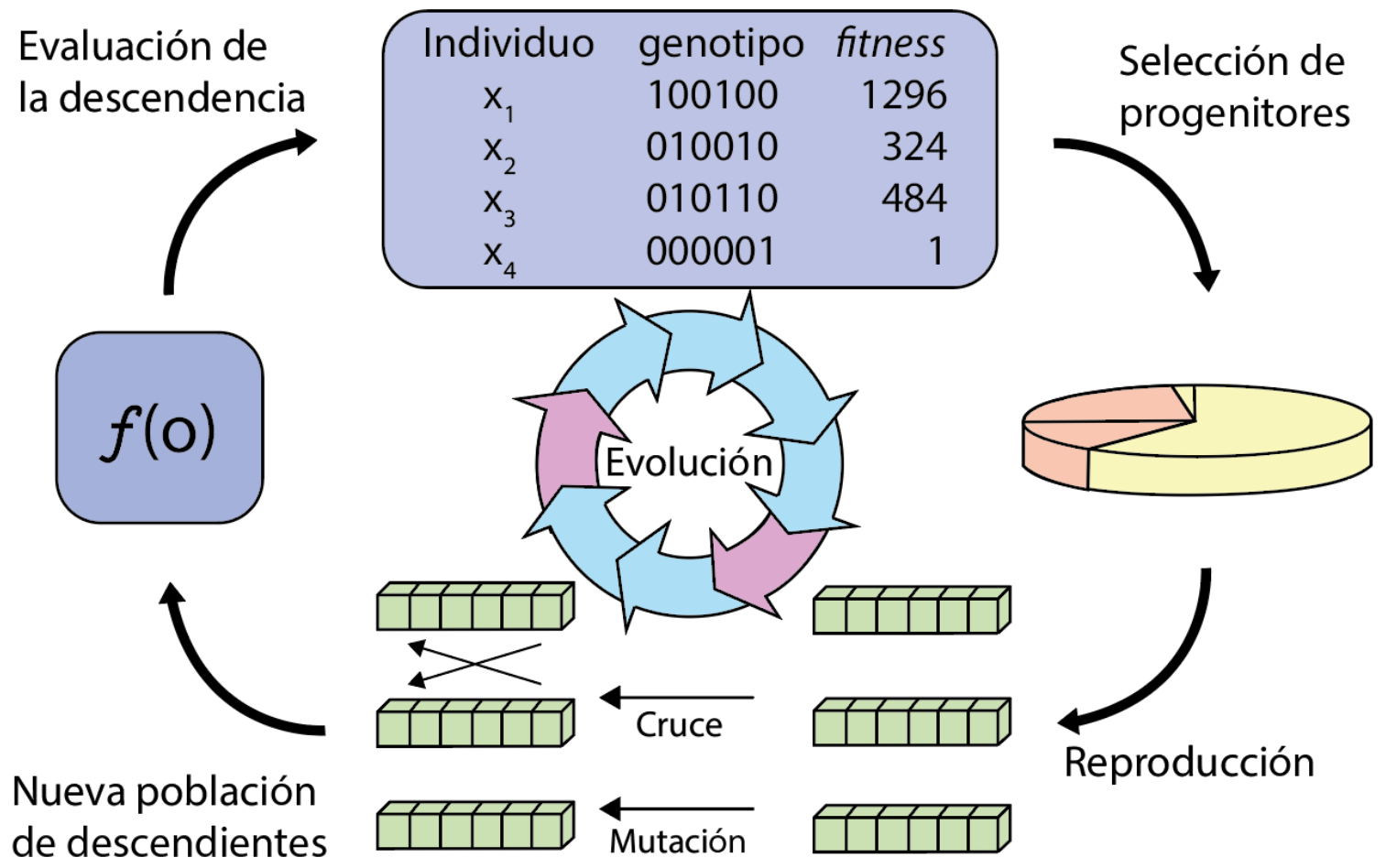

Figura 1. Diagrama del algoritmo genético

Asimismo, los algoritmos de interpolación. MatLab, por ejemplo, proporciona diferentes funciones para interpolar datos con los distintos algoritmos existentes y sirve 
en la cotidianidad para estimar precios en función de las características de determinado producto. Se expresa de la siguiente forma:

$$
\operatorname{precio}(x)=\sum_{i \in \text { producto }}\left(\operatorname{precio}(x i) * \frac{\operatorname{distancia}(x, x i)}{\sum_{j \in \text { producto }} \operatorname{disctancia}(x, x j)}\right)
$$

donde se asume que distancia $(a, b)$ es la distancia que hay desde el producto a hasta el producto $\mathrm{b}$, definidos por los valores de sus atributos $(a 1, \ldots$, an y $b 1, \ldots, b n$ respectivamente.

$$
\operatorname{distancia}(a, b)=\sqrt{\left|a_{1}-b_{1}\right|^{2}+\left|a_{2}-b_{2}\right|^{2}+\left|a_{3}-b_{3}\right|^{2}+\cdots+\left|a_{n}-b_{n}\right|^{2}}
$$

También se pueden mencionar algoritmos de agrupamiento. Estos son interesantes ya que pueden ayudar a clasificar los usuarios y clientes.

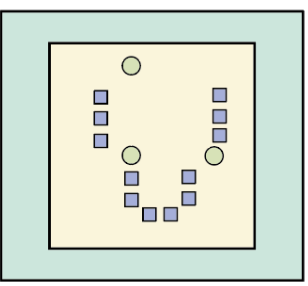

1) $\mathrm{k}$ centroides iniciales (en este caso $\mathrm{k}=3$ )

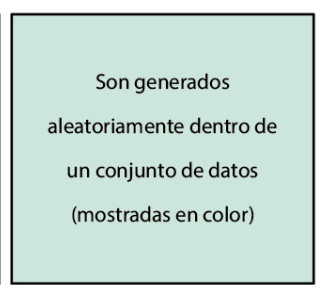

2) k grupos son generados asociandole el punto

Demostración del algoritmo estandar

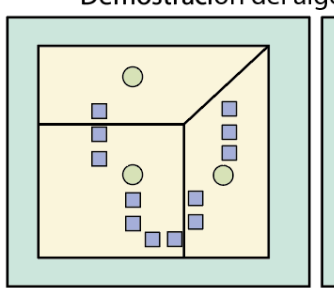

3) EL contenido de cada uno de los k grupos se recalcula

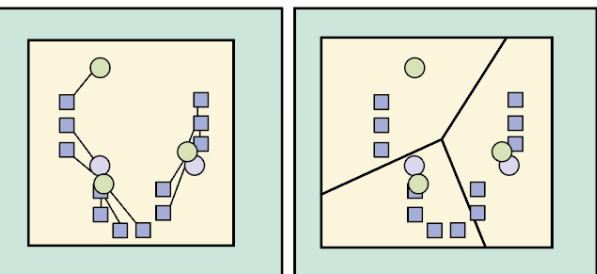

4) Pasos 2 y 3 se repiten hasta que se logre la convergencia

Figura 2. Diagrama Algoritmo de Agrupamiento K-MEANS

Además, las matemáticas se pueden manifestar mediante fórmulas para el estudio de mercado, punto de equilibrio, ingresos y costos, entre otros elementos que entran en juego en el marketing.

Tomando en cuenta, así, la relevancia del punto de equilibrio para tomar decisiones en las actividades de un negocio, se utiliza este análisis cuyos resultados son claves para tomar decisiones sobre el funcionamiento de toda empresa (Mazón et al. 2017). El punto de equilibrio es aquel volumen de ventas donde los ingresos totales se igualan a los costos totales, en este punto la empresa no gana ni pierde (Vallejos \& Chiliquinga, 2017). Para el cálculo del punto de equilibrio se hace uso de los siguientes términos: 
Fórmula para calcular punto de equilibrio:

$\left(\mathrm{P}^{*} \mathrm{Q}\right)-\left(\mathrm{CV}^{*} \mathrm{Q}\right)-\mathrm{CFT}=0 \mathrm{Q}^{*}(\mathrm{P}-\mathrm{CV})=\mathrm{CFT} \mathrm{Q}=\mathrm{CFT} /(\mathrm{P}-\mathrm{CV})=\mathrm{CFT} / \mathrm{MCU} \mathrm{Q}=$

\section{$\mathrm{CFT} / \mathrm{MCU}$}

\section{$\mathrm{Qe}=\mathrm{CFT} / \mathrm{MCU}$}

$\mathrm{P}=$ precio de venta del producto

$\mathrm{CV}=$ costo variable por producto

$\mathrm{CFT}=\operatorname{costos}$ fijos totales

$\mathrm{Q}=$ volumen de ventas del producto

$\mathrm{MCU}=$ margen de contribución unitario

$\mathrm{Qe}=$ volumen de ventas del producto cuando la utilidad es cero

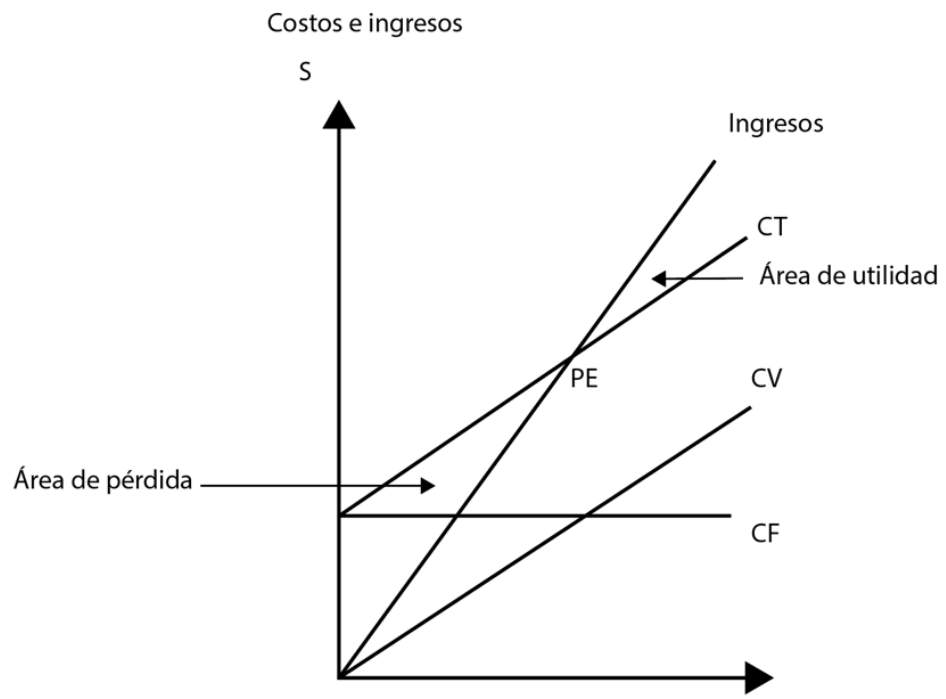

Figura 3. Gráfico ilustrativo del Punto de Equilibrio

Entre las fórmulas utilizadas con el punto de equilibrio se tienen:

\begin{tabular}{|l|l|}
\hline Punto de equilibrio & $\left(\mathrm{P}^{*} \mathrm{Q}\right)-(\mathrm{CV} * \mathrm{Q})-\mathrm{CFT}=0$ \\
\hline Costos fijos totales & $\mathrm{Q} *(\mathrm{P}-\mathrm{CV})=\mathrm{CFT}$ \\
\hline Volumen de ventas del producto & $\mathrm{Q}=\mathrm{CFT} /(\mathrm{P}-\mathrm{CV})=\mathrm{CFT} / \mathrm{MCU}$ \\
\hline Volumen de ventas del producto & $\mathrm{Q}=\mathrm{CFT} / \mathrm{MCU}$ \\
\hline $\begin{array}{l}\text { Volumen de ventas del producto } \\
\text { cuando la utilidad es cero }\end{array}$ & $\mathrm{Qe}=\mathrm{CFT} / \mathrm{MCU}$ \\
\hline
\end{tabular}




\begin{tabular}{|c|c|}
\hline En función de ingreso & $\begin{array}{l}\qquad \mathrm{PE}=\text { Punto de equilibrio en } \\
\text { unidades monetarias / Precio de venta } \\
\text { unitario }\end{array}$ \\
\hline $\begin{array}{l}\text { En función de ingreso, en unidades } \\
\text { monetarias }\end{array}$ & $\begin{array}{l}\qquad \mathrm{PE}=\mathrm{CF}+((\mathrm{CV} / \text { ventas }) * \mathrm{PE})+ \\
\text { utilidad }\end{array}$ \\
\hline $\begin{array}{l}\text { En base a la formula del método de } \\
\text { la ecuación }\end{array}$ & $\mathrm{Pvu} * \mathrm{Q}=\mathrm{CF}+\mathrm{Cvu} * \mathrm{Q}+\mathrm{Ut}$ \\
\hline $\begin{array}{l}\text { En base de la ecuación de } \\
\text { contribución }\end{array}$ & $\mathrm{PE}=\mathrm{CF} / \% \mathrm{M}$ \\
\hline $\begin{array}{l}\text { En base de la ecuación de } \\
\text { contribución, en unidades monetarias }\end{array}$ & $\mathrm{PE}=\mathrm{CF} / 1-(\mathrm{CV} / \mathrm{V})$ \\
\hline $\begin{array}{c}\text { En función del margen de } \\
\text { contribución (unidades de producto) }\end{array}$ & $\mathrm{PE}=\mathrm{CF} / \mathrm{Pvu}-\mathrm{CVu}$ \\
\hline $\begin{array}{l}\text { En función del margen de } \\
\text { contribución (unidades monetarias) }\end{array}$ & $\begin{array}{l}\mathrm{PE}=\text { Punto de equilibrio en } \\
\text { volumen } * \mathrm{PVu}\end{array}$ \\
\hline Umbral de rentabilidad & $\mathrm{UR}=(\mathrm{Pvu} * \mathrm{CF}) /(\mathrm{Pvu}-\mathrm{CVu})$ \\
\hline Margen de seguridad & $\begin{array}{l}\qquad \mathrm{MS}=(\text { ventas totales }- \text { ventas en } \\
\text { punto de equilibrio / ventas totales })\end{array}$ \\
\hline
\end{tabular}

Figura 4. Fórmulas con el punto de equilibrio

En un uso más genérico, las matemáticas en el marketing también se hacen presentes a través de los métodos de encuestas. Esta, es una técnica destinada a obtener información sobre la preferencia del consumidor con relación a un determinado producto no tradicional (nuevo) o de un producto que ya existe en el Mercado, pero del cual no se tiene información estadística alguna. Para ello, se tiene la siguiente fórmula:

$$
n=\frac{N * K_{\frac{a}{2}}^{2} * p * q}{N * \in^{2}+K_{\frac{a}{2}} * p * q}
$$

Donde:

\section{$\eta$ : Muestra o numero de encuestas}

\section{$N$ : Población}

$K_{\frac{a}{2}}:$ Valor de la tabla Normal para un nivel de significancia $\left(\right.$ si $\alpha=5 \%=>K_{\frac{\alpha}{2}}$

$$
=1.96)
$$


$p=$ Probabilidad éxito $(p=0.50)$

$q=$ Probabilidad fracaso $(q=0.50)$

$\varepsilon=$ Error absoluto de la Muestra del 2\% al 6\%

Así también se pueden mencionar fórmulas respecto a la elasticidad de la demanda, la cual tiene que ver con la elasticidad precio de la Demanda que mide el grado al cual los consumidores responden ante los cambios del precio del producto.

$$
E_{p}=\frac{\% \Delta \text { en la cantidad demandada }}{\% \Delta \text { en el precio }}
$$

Además, sobre la demanda también se pueden hacer proyecciones a través de fórmulas matemáticas.

\begin{tabular}{|c|c|c|}
\hline Proy. Lineal & $Y=A+B X$ & \\
\hline Proy. Exponencial & $Y=\alpha \varrho^{3 x}$ \\
\hline Proy. Potencial & $Y=a x^{3}$ & \\
\hline Proy. Logaritmica & $Y=a+b \ln x$ & \\
\hline
\end{tabular}

Figura 5. Proyección de la Demanda por medio de tazas

\section{Discusión}

De acuerdo con la exploración documental realizada, es preciso destacar que las investigaciones desarrolladas en torno al campo de la estadística y su relación con la preparación profesional de las personas apuntan a la relevancia que tiene el desarrollo de estas destrezas y facultades para un buen desempeño laboral en cualquier espacio de trabajo. Por otro lado, a aplicación de los métodos estadísticos en el área de marketing ha descansado en asuntos académicos, en su mayoría, más allá de dedicarse al abordaje práctico de la implementación de los planes organizacionales.

En efecto, la empleabilidad de la estadística subyace en una amplia gama de espacios académicos que van desde el estudio de distribuciones de frecuencias, gráficos, porcentajes e histogramas hasta el análisis de estadísticos descriptivos que permitan 
comprender el comportamiento de ciertas variables del mercado, para concluir en una elección de decisiones en la organización a través del análisis de las tablas de contingencia que representan la multiplicidad de variables involucradas en el análisis.

Sin embargo, a la estadística aplicada al estudio de mercados se hace desde el plano inferencial ya que en base a un universo se recolectan datos sobre una muestra que puede arrojar resultados que determinan las características de la población en general, como una ley universal del comportamiento de los hallazgos derivados de las investigaciones, no obstante, Leiva (2020) sostiene que dichas inferencias no siempre se ajustan a la rigurosidad metodológica que amerita un estudio de mercado. Por ello, debe tomarse con cuidado la acción de tomar decisiones que debe estar sustentada en el uso correcto y adecuado de las herramientas matemáticas en los abordajes de los mercados objetivos en una empresa.

En esta medida, contar con información precisa es un aval de competitividad ante las empresas que juegan en el mismo campo de mercado, no obstante, para las organizaciones es un reto tener entre sus filas personas con aptitudes y habilidades que le permitan realizar los análisis idóneos para cada caso. En todo caso, el proceso de recolección de datos y el análisis de los resultados, es una estrategia que debe presentarse de forma clara, concreta y sencilla de tal manera que lleve a dilucidar sobre las decisiones más pertinentes para la empresa.

\section{Conclusiones}

La mayoría de las decisiones empresariales son sometidas al estudio cuantitativo de las oportunidades que se presentan en los mercados. Las empresas que no han capacitado a su personal en el estudio de herramientas estadísticas, siempre van a presentar dificultades para poder implementar estudios de mercado óptimos a las necesidades de la organización.

El análisis cuantitativo no es la solución a los problemas empresariales, no obstante, la estadística puede dar el soporte para que las decisiones estén declaradas sobre bases de mayor solidez, lo cual representa un elemento positivo en el ámbito de la competitividad.

El análisis estadístico debe responder a un conglomerado de elementos involucrados que no deben estudiarse por separado son que deben ser vistos integralmente para ofrecer una visión más clara de la situación empresarial. 
Por otra parte, es necesario destacar que la aplicación de herramientas estadísticas debe hacer de forma adecuada en el plano metodológico, de forma tal que ofrezca resultados de mayor validez a la situación en estudio. El respeto por el sustento teórico en estadística es una ventaja para la aplicación práctica en los estudios de mercado.

El presente estudio emplea de manera sencilla elementos que incentivas el so de las herramientas estadísticas en el marketing, considerando que los análisis de datos son un instrumento valioso para el sector ejecutivo al momento de tomar decisiones.

\section{Referencias}

American Marketing Association (2008). The American Marketing Association Releases New Definition for Marketing. https://bit.ly/3a3tgSk

Baines, P., Fill, C. \& Page, K. (2011). Marketing. New York: Oxford University Press Inc.,

Barney, J.B. (2002). Gaining and sustaining competitive advantage. New Jersey: Prentice Hall.

Baker, M.J. \& Hart, S.J. (ed.) (2008). The Marketing Book. Bunlington, USA: Elsevier Ltd.

Bartels, R. (1988). The History of Marketing Thought. Columbus: Publishing Horizons Inc.

Dann, S. (2020). Redefining social marketing with contemporary. Commercial marketing definitions. Journal of Business Research, 63(2), 147-153.

Gómez, L (2013) Diseño de un sistema de información de marketing. (Tesis para optar por el título de Master en Dirección) Universidad de La Habana. Los sistemas de Marketing, http://cvb.ehu.es/open_course_ware/castellano/ social_juri/marketing/tema5/tema-5.pdf

González, E.; López, M.J. \& Otero, M.C. (2014). Manual práctico de marketing. Madrid: Pearson.

Hernández, M., \& Rodríguez, A. (2003). El objeto de estudio de la dicisplina marketing. Estudios Gerenciales, 19(87), 67-91.

Jobber, D. (2007). Principles and Practice of Marketing. Berkshire: mcgraw-Hill Education.

Leiva, D. (2020). La importancia de la estadística inferencial en las empresas. https:/www.clubensayos.com/Negocios/LA-IMPORTANCIA-DE-LAESTAD\%C3\%8DSTICA-INFERENCIAL-EN-LAS/5158693.html 
Kotler, P.T. \& Keller, K.L. (2013). Dirección de marketing. Madrid: Pearson.

Kotler, P.; Keller, K. L.; Brady, M.; Goodman, M. \& Hansen, T. (2009). Marketing Management. Essex: Pearson Education.

Mazón, L., Villao, D., Núñez, W. \& Serrano, M. (2017) Análisis de punto de equilibrio en la toma de decisiones de un negocio: caso Grand Bazar Riobamba-Ecuador. Revista de Estrategias del Desarrollo Empresarial, Vol. 3, N8, pp. 14-24.

Porter, M.E. (2002). Ventaja Competitiva. Barcelona: Alay Ediciones.

Vallejos, H., \& Chiliquinga, M. (2017). Costos-Modalidad órdenes de producción. Ibarra - Ecuador: Universidad Técnica del Norte.

Villegas, D. (2019). La importancia de la estadística aplicada para la toma de decisiones en Marketing. Revista Investigación y Negocios. http://www.scielo.org.bo/scielo.php?script=sci arttext\&pid=S252127372019000200004

Westwood, J. (2013). How to write a marketing plan. (4th ed.) London: Kogan Page. 